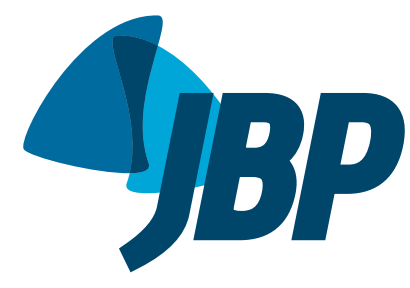

1. Multimagem Diagnósticos, Goiânia (GO) Brasil.

2. Universidade Federal de Goiás, Goiânia (GO) Brasil.

3. Grupo Fleury, São Paulo (SP) Brasil.

4. Universidade Federal do Rio de Janeiro, Rio de Janeiro (RJ) Brasil.

Submitted: 4 December 2016.

Accepted: 4 May 2017

Study carried out at Multimagem Diagnósticos and at the Universidade Federal de Goiás, Goiânia (GO) Brasil.

\section{Usual interstitial pneumonia: typical, possible, and "inconsistent" patterns}

\author{
Pedro Paulo Teixeira e Silva Torres ${ }^{1}$, Marcelo Fouad Rabahi², \\ Maria Auxiliadora Carmo Moreira², Gustavo de Souza Portes Meirelles ${ }^{3}$, \\ Edson Marchiori ${ }^{4}$
}

\begin{abstract}
Idiopathic pulmonary fibrosis is a severe and progressive chronic fibrosing interstitial lung disease, a definitive diagnosis being established by specific combinations of clinical, radiological, and pathological findings. According to current international guidelines, HRCT plays a key role in establishing a diagnosis of usual interstitial pneumonia (UIP). Current guidelines describe three UIP patterns based on HRCT findings: a typical UIP pattern; a pattern designated "possible UIP"; and a pattern designated "inconsistent with UIP", each pattern having important diagnostic implications. A typical UIP pattern on HRCT is highly accurate for the presence of histopathological UIP, being currently considered to be diagnostic of UIP. The remaining patterns require further diagnostic investigation. Other known causes of a UIP pattern include drug-induced interstitial lung disease, chronic hypersensitivity pneumonitis, occupational diseases (e.g., asbestosis), and connective tissue diseases, all of which should be included in the clinical differential diagnosis. Given the importance of CT studies in establishing a diagnosis and the possibility of interobserver variability, the objective of this pictorial essay was to illustrate all three UIP patterns on HRCT.
\end{abstract}

Keywords: Tomography, X-ray computed; Lung diseases, interstitial; Pulmonary fibrosis.

\section{INTRODUCTION}

Idiopathic pulmonary fibrosis (IPF) is a chronic fibrosing interstitial lung disease that is usually progressive. Recently defined diagnostic criteria include exclusion of other known causes of interstitial lung disease, the presence of a usual interstitial pneumonia (UIP) pattern on HRCT, and specific combinations of HRCT and surgical lung biopsy patterns. ${ }^{(1,2)}$

A typical UIP pattern on HRCT has been shown to be highly accurate for the presence of a UIP pattern on surgical lung biopsy (90-100\%); reliable imaging features of UIP are currently considered to be diagnostic of a UIP pattern, without the need for a surgical lung biopsy. ${ }^{(1)}$ Surgical lung biopsy is currently recommended when HRCT findings are not typical of UIP, a definitive diagnosis being established by specific combinations of imaging and histopathological findings. (1) Therefore, correct interpretation of HRCT findings is essential for a definitive diagnosis, particularly in view of the fact that antifibrotic agents have recently been approved for use in the treatment of IPF. ${ }^{(3)}$

Current guidelines describe three UIP patterns based on HRCT findings: a typical UIP pattern (which eliminates the need for surgical lung biopsy); a pattern designated "possible UIP"; and a pattern designated "inconsistent with UIP", surgical lung biopsy being required in patients presenting with either of the last two patterns..$^{(1,2,4)}$ The objective of the present study was to describe and illustrate the criteria for classifying patients as having a typical UIP pattern, a possible UIP pattern, or an inconsistent with UIP pattern.

\section{HRCT FEATURES CHARACTERIZING UIP PATTERNS}

\section{Typical UIP pattern}

A typical UIP pattern on HRCT consists of predominantly basal and peripheral reticular opacities and honeycombing, with or without traction bronchiolectasis. In addition, all of the findings that are considered to be inconsistent with UIP must be absent (Figure 1). (1) When all of the aforementioned criteria are met, the findings are considered to be pathognomonic for UIP, eliminating the need for a surgical lung biopsy. ${ }^{(1)}$ There is good interobserver agreement among radiologists for typical UIP findings. ${ }^{(5,6)}$ It is of note that UIP and IPF are not synonyms, known causes of a UIP pattern including drug-induced interstitial lung disease, occupational diseases (e.g., asbestosis), hypersensitivity pneumonitis, and connective tissue diseases. ${ }^{(5)}$

\section{Possible UIP pattern}

A possible UIP pattern consists of predominantly basal and peripheral reticular opacities and no honeycombing or any of the findings that are considered to be inconsistent with UIP (Figure 2). ${ }^{(1)}$ A possible UIP pattern is less specific for UIP than is a typical UIP pattern, the main 
differential diagnosis being with fibrotic nonspecific interstitial pneumonia (NSIP). ${ }^{(5)}$ No honeycombing, extensive ground-glass opacity, subpleural sparing, and lower lobe volume loss are suggestive of NSIP. ${ }^{(7-9)}$ Honeycombing is rare in cases of NSIP, having been found in less than $5 \%$ of the patients with idiopathic NSIP investigated in one study. ${ }^{(7)}$

\section{Inconsistent with UIP pattern}

Findings that are considered to be inconsistent with UIP include a) longitudinal disease distribution in the middle and upper lung fields; b) peribronchovascular predominance of changes in the axial axis (Figure 3); c) extensive ground-glass opacity, the extent of which is greater than that of reticular opacities; d) bilateral scattered micronodules predominantly in the upper lung fields (Figure 4); e) cysts (multiple, bilateral, away from areas of fibrosis); f) a mosaic perfusion pattern/air trapping (bilateral, in three or more lobes; Figure 5); and g) consolidations. Several of the aforementioned findings are suggestive of chronic hypersensitivity pneumonitis (CHP), further investigation being required for a differential diagnosis. ${ }^{(5)}$

\section{SPECIAL CONSIDERATIONS}

\section{Technical aspects}

Technically satisfactory image acquisition is required for a correct diagnosis, minimum technical requirements including a) images acquired at full inhalation, without motion artifacts; b) thin, sequential or volumetric axial images with a reconstruction interval $\leq 2 \mathrm{~cm}$; c) slice thickness $\leq 2 \mathrm{~mm}$; d) use of a high-resolution algorithm; e) a field of view optimized to include only lung parenchyma; f) images acquired during exhalation are useful for defining air trapping; g) use of the prone position in case of uncertainty regarding position-dependent opacities; and $h$ ) use of multiplanar reconstructions of volume acquisition CT images. ${ }^{(10)}$

Inadequately performed inspiratory maneuvers can result in increased/heterogeneous lung attenuation and motion artifacts that can adversely affect CT studies (Figure 6). Suggestions for improving the quality of CT studies include the use of simple, clear instructions on how to perform inspiratory/expiratory maneuvers, patient training in different breathing levels before
(A)

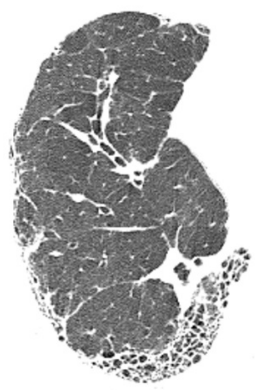

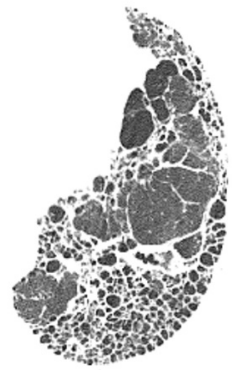

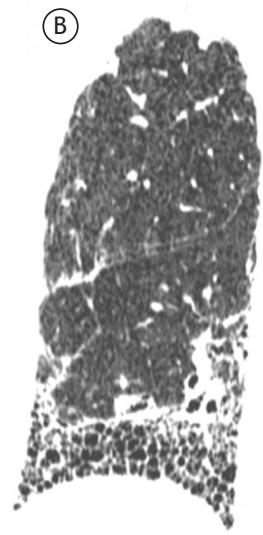

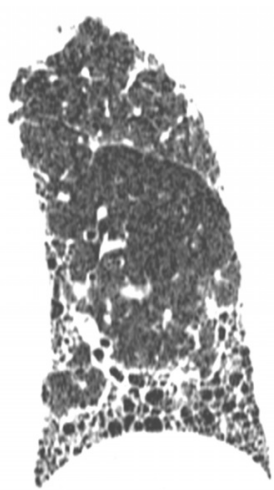

Figure 1. A 77-year-old female patient presenting with a typical usual interstitial pneumonia pattern. In A, axial CT scans of the chest with lung window settings, showing reticular opacities, traction bronchiectasis, and extensive honeycombing. In B, coronal reformatted CT images showing an apicobasal gradient of involvement.

(A)

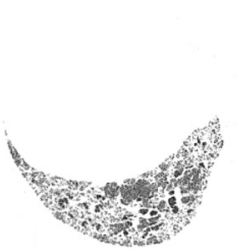

(B)

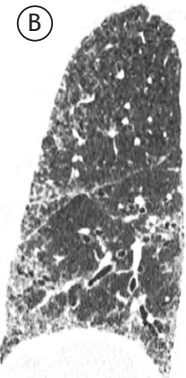

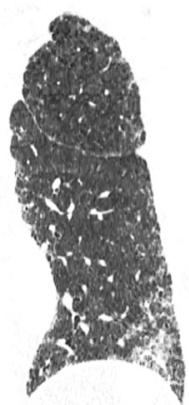

Figure 2. A 75-year-old male patient presenting with a possible usual interstitial pneumonia pattern. Axial CT scan of the chest with lung window settings (in A) and coronal reformatted CT image (in B) showing peripheral reticular opacities and traction bronchiolectasis (in A) and an apicobasal gradient (in B), without honeycombing. 

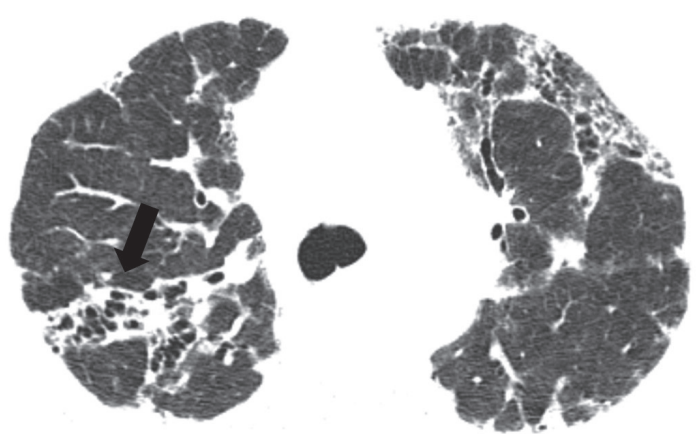

Figure 3. A 38-year-old female patient presenting with an inconsistent with usual interstitial pneumonia pattern and diagnosed with chronic hypersensitivity pneumonitis. Axial HRCT scan of the chest with lung window settings, showing diffuse reticular opacities with traction bronchiolectasis. Note severe peribronchovascular bundle involvement in the right upper lobe (arrow).
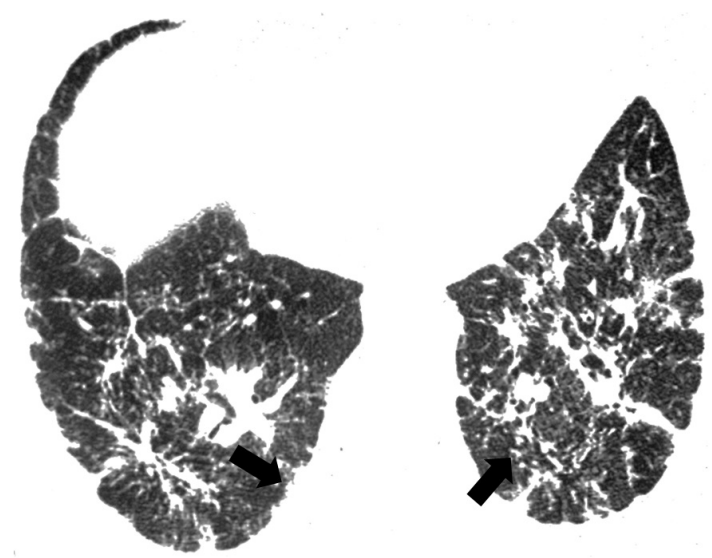

Figure 4. A 63-year-old female patient presenting with an inconsistent with usual interstitial pneumonia pattern and diagnosed with sarcoidosis. Axial HRCT scan of the chest with lung window settings, showing confluent, predominantly peribronchovascular reticular opacities with characteristics of micronodules with a perilymphatic distribution (arrows).

image acquisition, and the use of rest periods during sequential acquisitions. ${ }^{(10)}$

\section{Diagnosis of honeycombing}

A correct CT diagnosis of honeycombing is a crucial step in identifying a typical UIP pattern and establishing a clinical and imaging diagnosis of IPF. ${ }^{(1)}$ However, even among evaluators with extensive experience in interstitial lung disease, there is significant interobserver variability in attempts to detect honeycombing and differentiate it from other findings, such as traction bronchiectasis, cystic disease, and pulmonary emphysema (Figure 7).(11) Diagnostic criteria for honeycombing include predominantly subpleural cysts of $3-10 \mathrm{~mm}$ in diameter, sharing relatively thick (1-3 mm) walls and grouped on layers, and the exclusion of emphysema. ${ }^{(11,12)}$

\section{Interobserver agreement: CT criteria for UIP patterns}

Walsh et al. (13) evaluated interobserver agreement for the current criteria for a UIP pattern on CT.
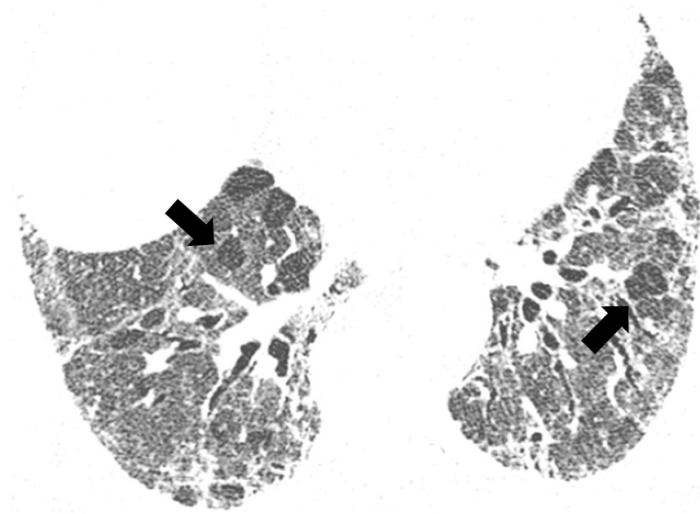

Figure 5. A 61-year-old female patient presenting with an inconsistent with usual interstitial pneumonia pattern and diagnosed with chronic hypersensitivity pneumonitis. Axial HRCT scans of the chest with lung window settings, showing diffuse reticular opacities, as well as areas of ground-glass attenuation associated with areas of decreased attenuation (arrows), characterizing a mosaic pattern.

Interobserver agreement was found to be only moderate for experienced general radiologists and thoracic radiologists, the difficulty in distinguishing among UIP patterns being attributed to discrepancies regarding the presence and distribution of honeycombing.

\section{Atypical patterns and differential diagnosis}

Although typical CT findings of UIP can predict a histopathological diagnosis of UIP, they are absent in up to $30 \%$ of patients. ${ }^{(14)}$ Sverzellati et al. ${ }^{(14)}$ studied histopathologically confirmed cases of UIP and found that radiologists made an alternative diagnosis in $62 \%$ of the cases. The aforementioned study ${ }^{(14)}$ shows that, although CT is highly accurate in diagnosing UIP in typical situations, CT studies should not be used in order to exclude the possibility of UIP. In atypical cases, first-choice diagnoses include NSIP, CHP, sarcoidosis, and chronic organizing pneumonia.

\section{Temporal evolution}

The clinical course of IPF is variable and unpredictable at the time of diagnosis; although most patients experience a slow progressive decline, some remain stable, whereas others experience a rapid decline. ${ }^{(1,15)}$ With regard to the severity of HRCT findings, areas of ground-glass attenuation usually progress to reticular opacities, which in turn progress to honeycombing, the extent of which increases over time. ${ }^{(16)}$ It is of note that CT interpretation changes over time, meaning that a possible UIP pattern can progress to a typical UIP pattern (Figure 8).

\section{Future directions}

The risks of performing a surgical lung biopsy in patients with interstitial lung disease should be taken into consideration; in many cases, diagnosis and treatment are delayed because patient clinical status is a contraindication to biopsy. ${ }^{(17,18)}$ Therefore, there is a growing interest in the noninvasive diagnosis of 

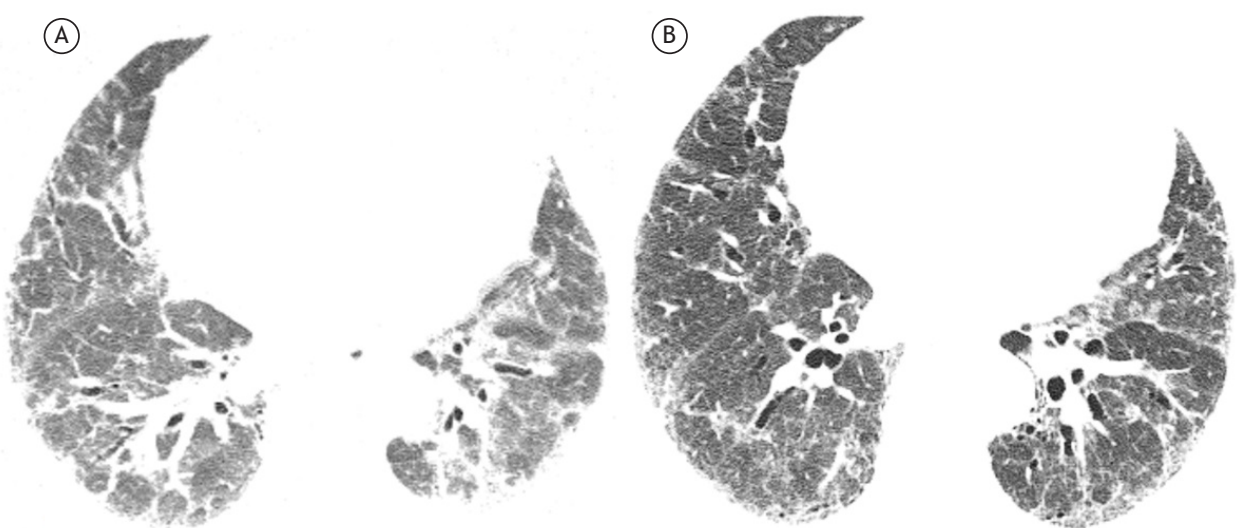

Figure 6. Axial CT scans of the chest with lung window settings. In A, scan taken during an inadequately performed inspiratory maneuver, the resulting image resembling diffuse ground-glass opacity. In B, a new scan, taken during an adequately performed inspiratory maneuver.
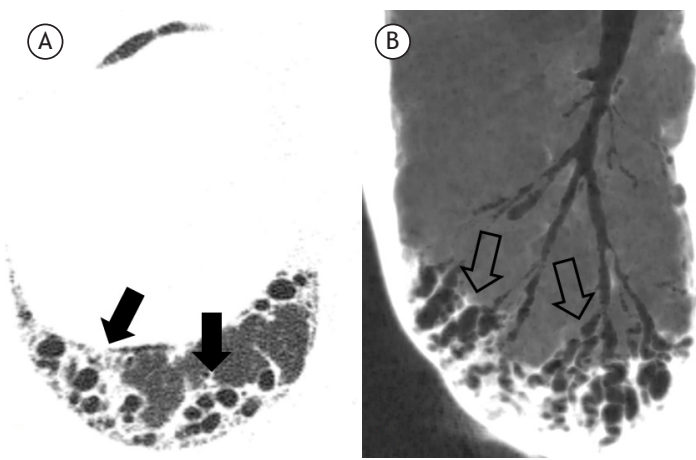

Figure 7. In $A$, axial HRCT scan of the chest with lung window settings. In $B$, coronal reformatted $C T$ image (minimum intensity projection). In $A$, images suggestive of a cluster of subpleural cysts (arrows), suspected of being honeycombing but found to be traction bronchiectasis (arrows) on oblique coronal reformatted CT images (in B).

IPF, particularly in cases of patients presenting with CT findings of possible UIP. Recent studies comparing IPF patients with a typical UIP pattern and those with a possible UIP pattern have shown clinical and functional similarities between the two groups of patients, as well as showing evidence of a comparable response to antifibrotic treatment with nintedanib. ${ }^{(19-21)}$ Several studies investigating patients with IPF have shown high rates of biopsy-proven UIP in those with a possible UIP pattern. ${ }^{(22-25)}$ Age at disease onset and the extent of fibrosis on initial HRCT scans have been significantly related to a high probability of IPF, a possible UIP pattern being suggestive of a clinical and radiological diagnosis of IPF in the following cases: a) typical clinical and demographic presentation (i.e., patients over 60 years of age presenting with dyspnea on exertion and pulmonary fibrosis of indeterminate etiology), as determined by a specialist in interstitial lung diseases; and $b$ ) imaging findings of possible UIP, according to a specialist in interstitial imaging. ${ }^{(24,26)}$

It is of note that some of the studies suggesting that CT findings of possible UIP are sufficient for a
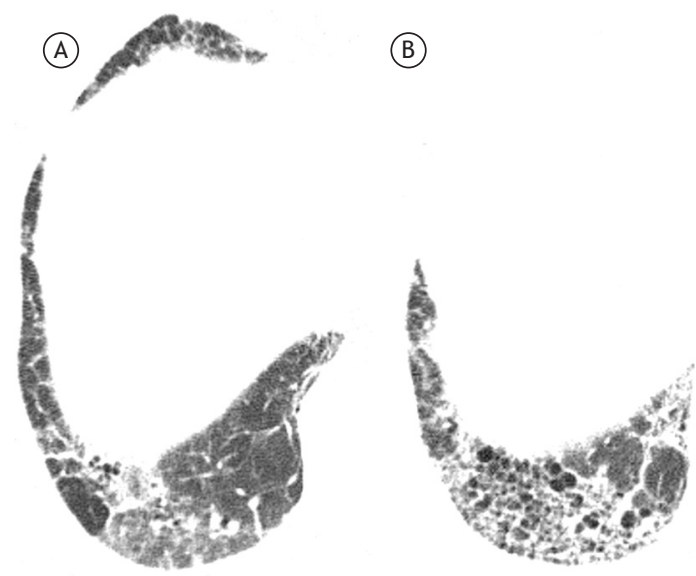

Figure 8. Axial HRCT scans of the chest with lung window settings, showing the right lower lobe. In A, initial CT findings meeting the criteria for possible usual interstitial pneumonia (UIP), i.e., reticular opacities and ground-glass attenuation, without honeycombing. In B, follow-up CT findings six years later, meeting the criteria for a typical UIP pattern, with disease progression and honeycombing.

diagnosis of IPF derived from clinical trials in which the prevalence of IPF was high, meaning that the results might have been overestimated. ${ }^{(23,24)}$ In a study conducted by Brownell et al., (25) it was found that a possible UIP pattern is highly specific for UIP on biopsy; however, the positive predictive value of that pattern is directly related to the prevalence of IPF in the study population. Therefore, according to the authors, a possible UIP pattern on HRCT should not be regarded as confirmatory of histopathological UIP in populations in whom the prevalence of IPF is low or indeterminate. ${ }^{(25)}$ Given that the prevalence of $\mathrm{CHP}$ is high (i.e., as high as 15\%) in Brazil, studies are needed in order to determine the prevalence of IPF in patients with possible UIP before a decision can be made regarding the need for biopsy in such patients. ${ }^{(27)}$

Noninvasive diagnostic algorithms for CHP have been proposed, including a typical CT pattern, lymphocytosis in BAL fluid (lymphocyte count > 20-30\%), and 
identification of a causal relationship; such algorithms are extremely useful in the diagnosis of fibrotic interstitial lung diseases, given that the differential diagnosis between CHP and IPF is often difficult. ${ }^{(27,28)}$

\section{FINAL CONSIDERATIONS}

Of all idiopathic interstitial lung diseases, IPF is the most common; it has a poor prognosis in most cases, and the histopathological substrate of IPF is UIP. (29)
In a recent review of the diagnostic algorithm for IPF, HRCT was shown to play an indispensable role in characterizing UIP, typical findings being diagnostic of UIP and atypical findings requiring histopathological analysis. ${ }^{(1)}$ For a definitive diagnosis, radiologists must be familiar with all UIP patterns and must be able to describe them accurately when writing radiological reports or participating in multidisciplinary meetings, particularly in view of current perspectives on the treatment of IPF, with the use of antifibrotic agents.

\section{REFERENCES}

1. Raghu G, Collard HR, Egan JJ, Martinez FJ, Behr J, Brown KK, et al. An official ATS/ERS/JRS/ALAT statement: idiopathic pulmonary fibrosis: evidence-based guidelines for diagnosis and management. Am J Respir Crit Care Med. 2011:183(6):788-824. https://doi. org/10.1164/rccm.2009-040GL

2. Baddini-Martinez J, Baldi BG, Costa CH, Jezler S, Lima MS, Rufino R Update on diagnosis and treatment of idiopathic pulmonary fibrosis. J Bras Pneumol. 2015;41(5):454-66. https://doi.org/10.1590/S180637132015000000152

3. Raghu G, Rochwerg B, Zhang Y, Garcia CA, Azuma A, Behr J, et al. An Official ATS/ERS/JRS/ALAT Clinical Practice Guideline: Treatment of Idiopathic Pulmonary Fibrosis. An Update of the 2011 Clinical Practice Guideline. Am J Respir Crit Care Med. 2015;192(2):e3-19. https://doi.org/10.1164/rccm.201506-1063ST

4. Travis WD, Costabel U, Hansell DM, King Jr TE, Lynch DA, Nicholson AG, et al. An official American Thoracic Society/European Respiratory Society statement: Update of the international multidisciplinary classification of the idiopathic interstitial pneumonias. Am J Respir Crit Care Med. 2013;188(6),733-48. https://doi.org/10.1164/rccm.201308-1483ST

5. Hodnett PA, Naidich DP. Fibrosing interstitial lung disease. A practical high-resolution computed tomography-based approach to diagnosis and management and a review of the literature. Am J Resp Crit Care Med. 2013;188(2):141-9. https://doi.org/10.1164/ rccm.201208-1544Cl

6. Flaherty KR, Thwaite EL, Kazerooni EA, Gross BH, Toews GB, Colby TV, et al. Radiological versus histological diagnosis in UIP and NSIP: survival implications. Thorax. 2003;58(2):143-8. https://doi. org/10.1136/thorax.58.2.143

7. Travis WD, Hunninghake G, King TE Jr, Lynch DA, Colby TV, Galvin $J R$, et al. Idiopathic nonspecific interstitial pneumonia: report of an American Thoracic Society project. Am J Respir Crit Care Med. 2008;177(12):1338-47. https://doi.org/10.1164/rccm.200611$16850 \mathrm{C}$

8. Silva $\mathrm{Cl}$, Müller NL, Lynch DA, Curran-Everett D, Brown KK, Lee KS, et al. Chronic hypersensitivity pneumonitis: differentiation from idiopathic pulmonary fibrosis and nonspecific interstitial pneumonia by using thin-section CT. Radiology. 2008;246(1):288-97. https:// doi.org/10.1148/radiol.2453061881

9. Sumikawa H, Jonkoh T, Fujimoto K, Arakawa H, Colby TV, Fukuoka $J$, et al. Pathologically proved nonspecific interstitial pneumonia: CT pattern analysis as compared with usual interstitial pneumonia CT pattern. Radiology. 2014;272(2):549-56. https://doi.org/10.1148/ radiol.14130853

10. Bankier AA, O'Donnell CR, Boiselle PM. Quality initiatives Respiratory instructions for CT examinations of the lungs: a hands-on guide. Radiographics. 2008;28(4):919-31. https://doi. org/10.1148/rg.284085035

11. Watadani T, Sakai F, Johkon T, Noma S, Akira M, Fujimoto K, et al. Interobserver variability in the $\mathrm{CT}$ assessment of honeycombing in the lungs. Radiology. 2013;266(3):936-44. https://doi.org/10.1148/ radiol. 12112516

12. Silva $\mathrm{Cl}$, Marchiori E, Souza Júnior AS, Müller NL; Comissão de Imagem da Sociedade Brasileira de Pneumologia e Tisiologia. Illustrated Brazilian consensus of terms and fundamental patterns in chest CT scans. J Bras Pneumol. 2010;36(1):99-123. https://doi. org/10.1590/S1806-37132010000100016

13. Walsh SL, Calandriello L, Sverzellati N, Wells AU, Hansell DM; UIP Observer Consort. Interobserver agreement for the ATS/ERS/JRS/ ALAT criteria for a UIP pattern on CT. Thorax. 2016;71(1):45-51. https://doi.org/10.1136/thoraxinl-2015-207252

14. Sverzellati N, Wells AU, Tomassetti S, Desai SR, Copley SJ, Aziz ZA, et al. Biopsy-proved idiopathic pulmonary fibrosis: spectrum of nondiagnostic thin-section CT diagnoses. Radiology. 2010;254(3):957-64. https://doi.org/10.1148/radiol.0990898

15. Raghu G. Idiopathic pulmonary fibrosis. A rational clinical approach Chest. 1987;92(1):148-54. https://doi.org/10.1378/chest.92.1.148

16. Misumi S, Lynch DA. Idiopathic pulmonary fibrosis/usual interstitial pneumonia: imaging diagnosis, spectrum of abnormalities, and temporal progression. Proc Am Thorac Soc. 2006;3(4):307-14. https://doi.org/10.1513/pats.200602-018TK

17. Hutchinson JP, Fogarty AW, McKeever TM, Hubbard RB. InHospital Mortality after Surgical Lung Biopsy for Interstitial Lung Disease in the United States. 2000 to 2011. Am J Resp Crit Care Med. 2016;193(10);1161-7. https://doi.org/10.1164/rccm.201508$16320 \mathrm{C}$

18. Hutchinson JP, McKeever TM, Fogarty AW, Navaratnam V Hubbard RB. Surgical lung biopsy for the diagnosis of interstitial lung disease in England: 1997-2008. Eur Respir J. 2016;48(5):14531461. https://doi.org/10.1183/13993003.00378-2016

19. Raghu G, Wells AU, Nicholson AG, Richeldi L, Flaherty KR, Le Maulf $F$, et al. Effect of Nintendanib in Subgroups of Idiopathic Pulmonary Fibrosis by Diagnostic Criteria. Am J Respir Crit Care Med. 2017;195(1):78-85. https://doi.org/10.1164/rccm.201602-04020C

20. Lee JW, Shehu E, Gjonbrataj J, Bahn YE, Rho BH, Lee MY, et al. Clinical Findings and outcomes in patients with possible usual interstitial pneumonia. Respir Med. 2015;109(4):510-6. https://doi. org/10.1016/j.rmed.2015.02.008

21. Gruden JF, Panse PM, Gotway MB, Jensen EA, Wellnitz CV Wesselius L. Diagnosis of Usual Interstitial Pneumonitis in the Absence of Honeycombing: Evaluation of Specific CT Criteria With Clinical Follow-Up in 38 Patients. AJR Am J Roentgenol. 2016;206(3):472-80. https://doi.org/10.2214/AJR.15.14525

22. Gruden JF, Panse PM, Leslie KO, Tazelaar HD, Colby TV. UIP diagnosed at surgical lung biopsy, 2000-2009: HRCT patterns and proposed classification system. AJR Am J Roentgenol. 2013;200(5):W458-67. https://doi.org/10.2214/AJR.12.9437

23. Chung JH, Chawla A, Pelito AL, Cool CD, Groshong SD, Talbert JL, et al. CT scan findings of probable usual interstitial pneumonitis have a high predictive value for histologic usual interstitial pneumonitis. Chest. 2015;147(2):450-459. https://doi.org/10.1378/chest.14-0976

24. Raghu G, Lynch D, Godwin JD, Webb R, Colby TV, Leslie KO, et al. Diagnosis of idiopathic pulmonary fibrosis with high-resolution CT in patients with little or no radiological evidence of honeycombing: secondary analysis of a randomised, controlled trial. Lancet Respir Med. 2014;2(4):277-84. https://doi.org/10.1016/S22132600(14)70011-6

25. Brownell R, Moua T, Henry TS, Elicker BM, White D, Vittinghoff $\mathrm{E}$, et al. The use of pretest probability increases the value of highresolution CT in diagnosing usual interstitial pneumonia. Thorax. 2017;72(5):424-429. https://doi.org/10.1136/thoraxjnl-2016-209671

26. Salisbury ML, Xia M, Murray S, Bartholmai BJ, Kazerooni EA, Meldrum CA, et al. Predictors of idiopathic pulmonary fibrosis in absence of radiologic honeycombing: A cross sectional analysis in ILD patients undergoing lung tissue sampling. Respir Med. 2016;118:88-95. https://doi.org/10.1016/j.rmed.2016.07.016

27. Pereira CA, Gimenez A, Kuranishi L, Storrer K. Chronic hypersensitivity pneumonitis. J Asthma Allergy. 2016:9:171-181. eCollection 2016. 
28. Salisbury ML, Myers JL, Belloli EA, Kazerooni EA, Martinez FJ, Flaherty KR. Diagnosis and Treatment of Fibrotic Hypersensitivity Pneumonia. Where We Stand and Where We Need to Go. Am J Respir Crit Care Med. 2016 Dec 21. [Epub ahead of print] https:// doi.org/10.1164/rccm.201608-1675PP

29. American Thoracic Society; European Respiratory Society. American
Thoracic Society/European Respiratory Society Internationa Multidisciplinary Consensus Classification of the Idiopathic Interstitial Pneumonias. This joint statement of the American Thoracic Society (ATS), and the European Respiratory Society (ERS) was adopted by the ATS board of directors, June 2001 and by the ERS Executive Committee, June 2001. Am J Respir Crit Care Med. 2002;165(2):277-304. https://doi.org/10.1164/ajrccm.165.2.ats01 